CLAWAR 2020: $23^{\text {rd }}$ International Conference on

Climbing and Walking Robots and the Support

Technologies for Mobile Machines,

Moscow, Russian Federation, 24-26 August 2020.

https://doi.org/10.13180/clawar.2020.24-26.08.34

\title{
VACUUM GENERATION FOR WALL CLIMBING ROBOT ADAPTIVE CONTACT TO SURFACE
}

\author{
V. G. GRADETSKY*, M. M. KNYAZKOV, E. A. SEMENOV, A. N. SUKHANOV and V. G. \\ CHASHCHUKHIN \\ Institute for Problems in Mechanics of the Russian Academy of Sciences Moscow, Russia $*$ E-mail: \\ gradet@ipmnet.ru \\ www.ipmnet.ru
}

\begin{abstract}
When mobile robots are moving vertically on complex ferromagnetic or non-ferromagnetic surfaces, such as ceilings, walls, inclined surfaces, contact with surfaces can be achieved by means of coupling devices that generate various levels of vacuum, which provide pressure using a vacuum pump. The controlled formation of vacuum makes it possible to adapt the contact to surfaces of various qualities and properties. The article discusses the processes of vacuum generation by means of a centrifugal pump and a turbine, as well as the action of a system developed on the basis of a contact due to a vacuum pump designed to carry out motion on vertical surfaces. The process of obtaining rarefied air in the chamber and the possibility of controlling the contacting force by changing the size of the gap between the surfaces of the movement of the robot and end of vacuum contact device are analyzed.

Experimental characteristics of pressure, separation force, and changes in the evacuation process are presented.
\end{abstract}

Keywords: vacuum pump, wall climbing robot, vacuum contact devices, recommendations for design.

\section{Introduction}

Many modern handling robots are equipped with vacuum grippers for products that differ in various shapes of gripping surfaces [1-5]. Mobile robots, in particular robots moving along complex surfaces, as well as surfaces located at different angles to the horizon, carry out fixing on surfaces using vacuum contact devices [6-16]. If the quality of the surfaces is not known in advance, then for the implementation of reliable adhesion there is a need for automatic recognition of surface properties and adaptation to them, i.e. application of methods of adaptation to surfaces. Despite the results achieved, the problem of adapting vacuum contact devices to surfaces with previously unknown properties has not yet been completely solved and requires the development of new approaches. One of them is based on the development of modeling methods for the interaction of vacuum contact devices with surfaces of various quality. A certain contribution in this direction is reflected in [17], which presents the results of the development of various models and the analysis of the use of modeling in order to evaluate the performance indicators of such contact devices. The most developed and used models are discussed: a simulation model of a robot holding system on a vertical surface, a model of a system with a vacuum contact. When creating models, the possibility of leakage in contact with the surface, the random distribution of cracks on the surface, and the ability to control the translational motion of the robot based on a finite state machine are taken into account. Attention is paid to the statistical modeling of the system of holding the robot on the surface and evaluating its effectiveness.

Methods of passive adaptation based on original design solutions of contact devices and the use of materials with the necessary elastic and wear-resistant properties and a multi-cell 
structure are developing. The prerequisites for the development of contact devices for robots of various scales with the properties of adapting to surfaces of previously unknown quality were noted earlier in many research results, for example, in [18-22]. Of particular interest are mobile robots designed to move on surfaces arbitrarily oriented in space. In these cases, it is necessary to use active and passive adaptation methods.

The article discusses the processes of creating a vacuum by means of a centrifugal pump and a turbine in order to adapt the robot to the surface along which the movement occurs.

\section{Some characteristics of the vortex flow in vacuum generation devices designed on the basis of a centrifugal pump or turbine.}

Let us consider in more detail the methods of holding the robot on a vertical surface using various vacuum generation devices. Figure 1 shows a mobile robot with vacuum pressing to the surface, based on the operation of a centrifugal pump. The movement of air flows between the robot body and the pressing surface is schematically represented by arrows. The gap X between the robot body and the motion surface may vary.

The vacuum contact device includes a pump and a robot body, the base of which forms an alternating pneumatic resistance with the displacement surface, the flow through which depends on the gap $\mathrm{x}$ and is absent when $\mathrm{x}$ is close to zero.

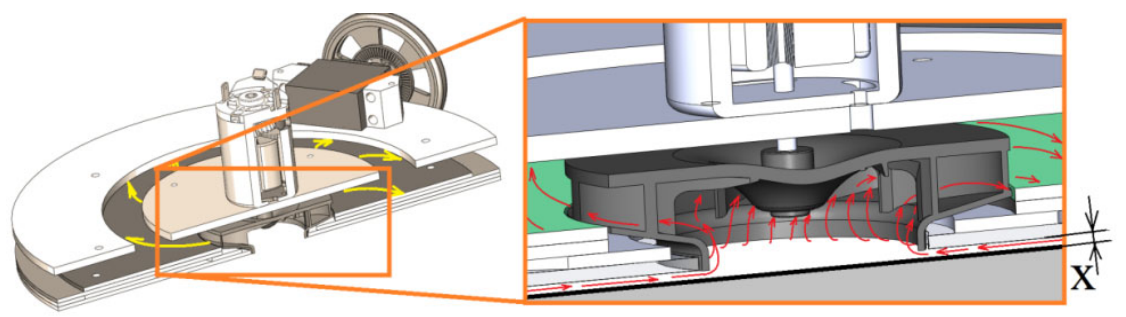

Figure 1 Mobile robot with a vacuum contact, operating on the basis of a centrifugal pump

Another design of the vacuum-contacted robot involves the use of a turbine (Figure 2). The design of this robot is similar to the design of a robot with a centrifugal pump with the exception of the vacuum generation device. The movement of air flows between the robot body and the pressing surface is schematically represented by arrows. In contrast to a centrifugal pump, air flows in this case pass through the turbine in direct flow.

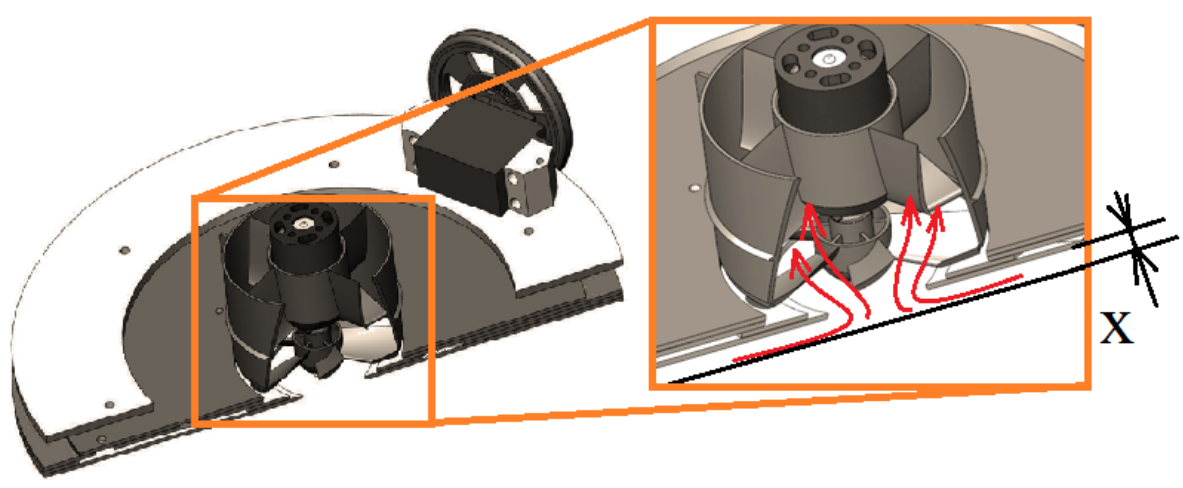

Figure 2 Mobile robot with vacuum pressing, working on the basis of a turbine

The full component of the flow velocity inside the vortex chamber can be expressed in terms of the radial and tangential components. The distribution of tangential velocities is usually expressed as degrees of dependence [23].

$$
\frac{w_{1}}{w_{2}}=\frac{v_{1}}{v_{2}}=\frac{R}{r}
$$


$\mathrm{w}_{1}, \mathrm{v}_{1}$ - angular and linear tangential flow rates,

$\mathrm{W}_{2}, \mathrm{~V}_{2}-$ angular and linear radial flow velocities,

$\mathrm{R}$ - the radius of the vortex chamber, $\mathrm{r}$ - is the variable radius of the resulting vortex flow.

Assume that the pressure gradient in the radial direction is expressed

$$
\frac{d p}{d r}=\rho \frac{v_{1}^{2}}{r}
$$

Given that $v_{1}=v_{2}\left(\frac{R}{r}\right)$, we get from the previous expression

$d p=\rho v_{2}^{2} \frac{R^{2}}{r^{3}} d r$

Integrating the last equation in the range from $\mathrm{r}_{2}$ to $\mathrm{R}$, we obtain

$$
\Delta p=\frac{\rho v_{2}^{2}}{2}\left[\left(\frac{R}{r_{2}}\right)^{2}-1\right]
$$

Outlet pressure:

$$
P_{2}=-\rho \frac{v_{2}^{2}}{2}\left[\left(\frac{R}{r_{2}}\right)^{2}-1\right]
$$

Consider the relationship between the parameters for a centrifugal pump and a turbine.

If the turbine power is spent only on creating a vacuum, then the pressure created by the turbine or centrifugal pump can be calculated by the formula:

$$
P=P_{1}+\frac{v^{2}}{2} \rho
$$

Here $\mathrm{P}_{1}-$ is the suction depression, $\mathrm{v}-$ is the air velocity at the outlet, $\rho$ - is the density under suction conditions depending on the gas constant, temperature, pressure, and humidity. The mass amount of air pumped out by the turbine is

$$
\mathrm{m}=\rho Q \text {, }
$$

where $\mathrm{Q}$ - is the mass flow rate.

The total pressure developed by the turbine depends on the shape factor of the blades $\varphi$, density $\rho$ and peripheral speed $\mathrm{U}_{2}$ at the exit of the impeller, corresponding to the outer circle where the ends of the blades are located.

$$
p=\varphi \cdot u_{2}^{2} \cdot \rho
$$

$\varphi=1,1 \div 0,5$, depending on the shape of the blades.

For forward curved blades, $\varphi=1,0 \div 1,1$.

For radial blades, $\varphi=0,9 \div 0,7$.

For downward curved blades, $\varphi=0,7 \div 0,5$.

For the ratio of the parameters of the centrifugal pump and the turbine, the law of proportionality is valid.

The pressure developed by a centrifugal pump or turbine is proportional to the square of the rotation speed, i.e. $P_{2}=\frac{w_{2}}{w_{1}} \cdot P_{1}$.

$$
\frac{P_{1}}{P_{2}}=\frac{w_{1}^{2}}{w_{2}^{2}} ; \quad \frac{P_{1}}{P_{2}}=\frac{\Pi_{1}^{2}}{\Pi_{2}^{2}}
$$

Pump performance is directly proportional to average speed.

$$
\frac{Q_{1}}{Q_{2}}=\frac{w_{1}}{w_{2}}, \quad Q_{2}=\frac{w_{1}}{w_{2}} Q_{1}
$$

$\mathrm{P}_{1}$ and $\mathrm{P}_{2}$ and $\mathrm{Q}_{1}$ and $\mathrm{Q}_{2}$ are respectively the pressure and flow at rotation speeds $\mathrm{w}_{1}$ and $\mathrm{W}_{2}$. The dependence of pressure on flow rate is expressed as follows:

$$
\frac{P_{1}}{P_{2}}=\frac{Q_{1}^{2}}{Q_{2}^{2}}=\text { const, }
$$

which allows you to determine the pressure, and therefore the pressure against the surface of the clutch.

Power is proportional to the cube of the speed of rotation of the wheel, i.e.

$$
\frac{N_{1}}{Q_{1}^{3}}=\frac{N_{2}}{Q_{2}^{3}}, \text { or } N_{2}=\left(\frac{Q_{2}}{Q_{1}}\right)^{3} \cdot N_{1}
$$


To calculate the operating mode in the system "centrifugal pump - clutch with the surface" or "turbine - clutch with the surface" it is necessary to jointly solve the equations of dependence of pressure on the flow rate of the pump or turbine and the clutch.

If in general terms the dependence of the pressure on the flow rate is known for the pump, and for the clutch device there is some nonlinear relationship between $\mathrm{P}$ and Q, which at the same time depends on the value of the gap $\mathrm{x}$, which can be found experimentally, then it is possible to graphically determine the operating point $\mathrm{A}$.

The type of pump performance and aerodynamic drag of the contacting device, pressure versus flow rate in relative coordinates are shown in Figure. 5, a, b. Here $P_{\mathrm{H}}, \mathrm{P}_{\mathrm{y}}$ and $\mathrm{Q}_{\mathrm{H}}, \mathrm{Q}_{\mathrm{y}}$ are the pressures and flows in the cavities of the pump and the chamber of the vacuum contacting device, respectively; $\mathrm{P}_{\text {но }}, \mathrm{R}_{\text {уо }}$ and $\mathrm{Q}_{\text {но }}, \mathrm{Q}_{\text {уо }}$ are the average values of pressures and flows in the cavity of the pump and chamber of the vacuum contacting device.

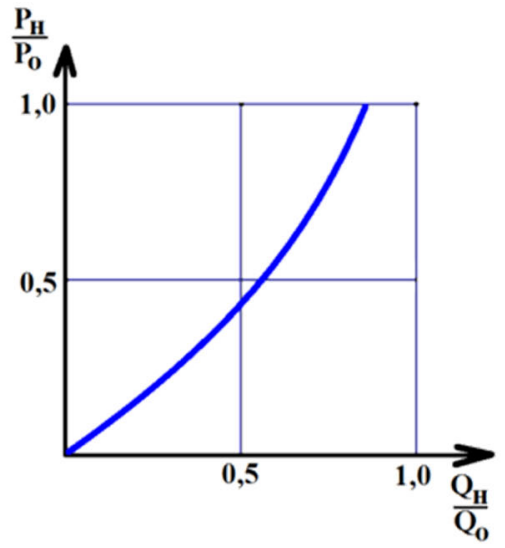

a)

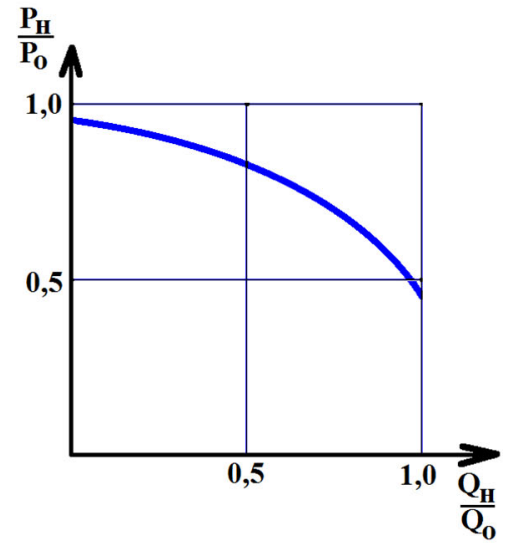

b)

Figure 5 Operating quality characteristics of the pump (a) and the chamber of the vacuum contacting device (b).

Finding point A of the operating mode is shown in Figure. 6, where the dependences of pressure on flow for pump 1 and aerodynamic resistance of the contacting device 2, 3 and 4 are combined. In this case, graph 2 corresponds to the nominal operating mode, and graphs 3, 4 show the deviation from the nominal mode. This point determines the supply of the required amount of air by this injection device into the system and the pressure developed thereby. Thus, the intersection point is the working point of the system under consideration. The inclusion of pressure feedback corresponds to the adaptive mode of operation when, as a result of controlled adaptation, schedules 3 and 4 come close to schedule 2, and their combination determines the completion of adaptation.

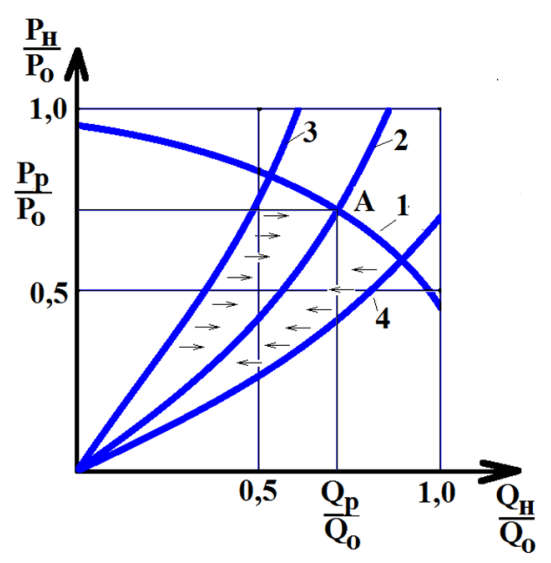


Figure 6 Finding the operating mode of the system "turbine or pump - pressure device to the surface", where it is indicated: 1 - pump characteristic, 2, 3,4 - resistance characteristic of the pressure device to the surface, A - operating point.

A similar current feedback determines the adaptation according to the force of the contact.

\section{An experimental study of creating a vacuum using a centrifugal pump and a turbine.}

To determine the rational characteristics of the gap X, the current consumption I, and the pressing force $\mathrm{F}$, experimental studies were carried out. Figure 7 shows the relationship $\mathrm{F}=\mathrm{f}(\mathrm{X})$.

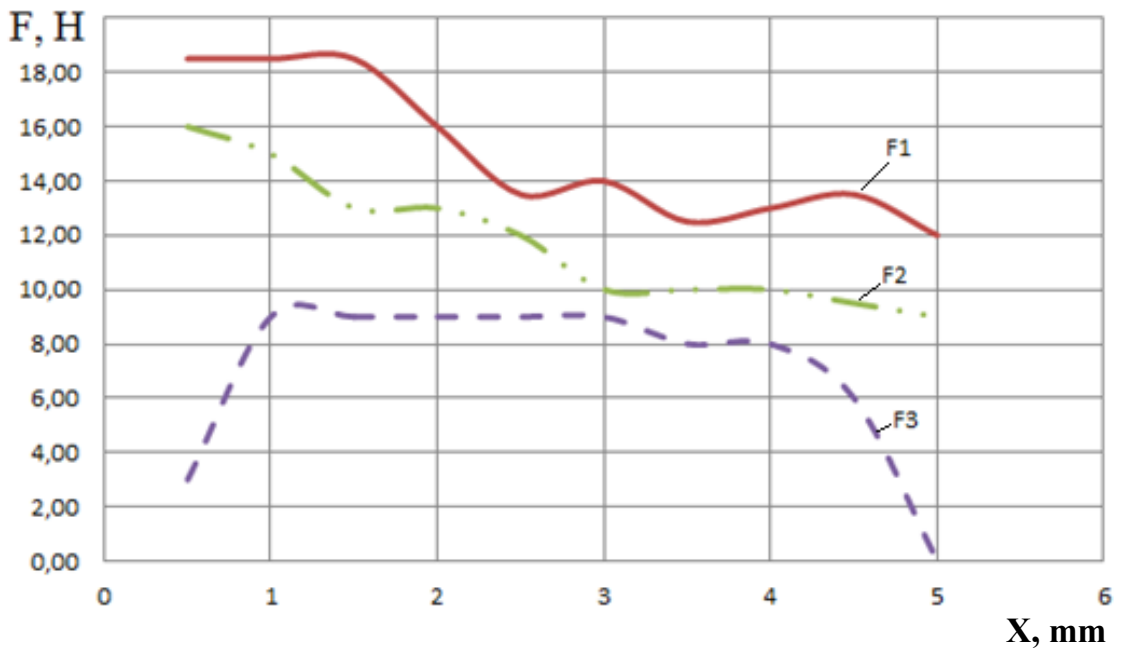

Figure 7 Dependence of the pressing force of the adhesion device to the surface on the gap value $\mathrm{x}$. F1 - high power centrifugal pump, F2 - centrifugal pump, F3 - turbine

According to the results of experimental studies, the effective value of the force of pressing the robot to the displacement surface from the gap $\mathrm{X}$ in the range from $0.5 \mathrm{~mm}$ to 5 $\mathrm{mm}$ was determined. As can be seen from the obtained characteristics, the force $\mathrm{F}$ for robots with a centrifugal pump took maximum values with a gap of $0.5 \mathrm{~mm}$. In experiments conducted with a turbine-based vacuum generation device, the force $\mathrm{F}$ took maximum values when the gap between the robot and the surface was $1 \mathrm{~mm}$.

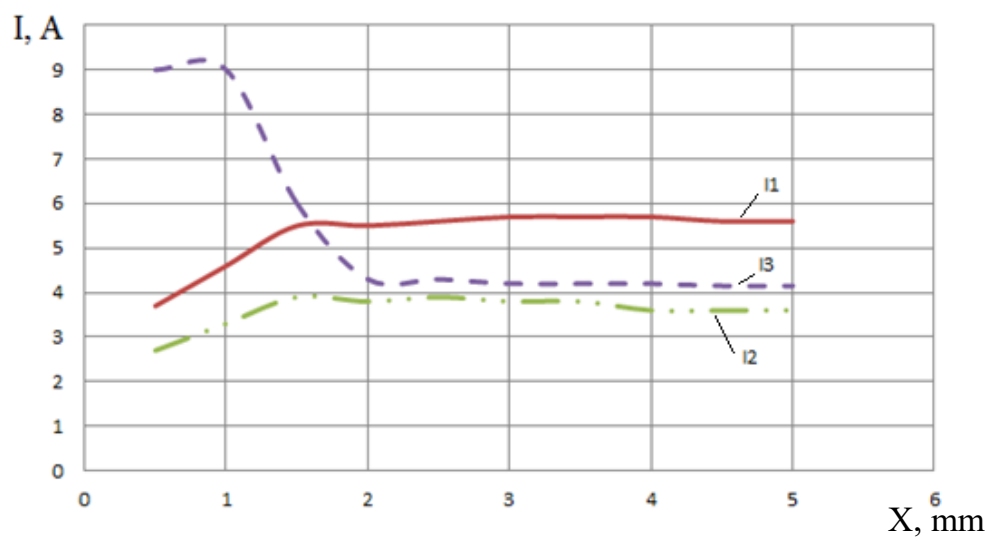

Figure. 8 Dependence of the current strength in the winding of the DC motor with independent excitation of the pressing device on the gap value. I1 - high power centrifugal pump, I2 - centrifugal pump, I3 - turbine

To minimize energy consumption, currents in the excitation windings of direct current motors (DC motors), which drive the structural elements of vacuum generation devices, were investigated. As can be seen from the results obtained (Figure 8), the lowest energy consumption 
by a robot with a centrifugal pump corresponds to a gap variation range from $0.5 \mathrm{~mm}$ to $1 \mathrm{~mm}$. The energy consumption for driving the turbine in the second type of robot showed a minimum current value with a gap of $2 \mathrm{~mm}$ to $5 \mathrm{~mm}$.

In the cavity of the vacuum contact device for coupling to surfaces, the absolute pressure P2 depends on the gap between the plane along which the robot moves and the end surface of the vacuum contact device, i.e. $\mathrm{P} 2=\mathrm{f}(\mathrm{x})$.

It should be borne in mind that the dependence $\mathrm{P} 2=\mathrm{f}(\mathrm{x})$ in the general case, nonlinear.

If $\mathrm{x} \geq \mathrm{xpr}$, then $\mathrm{P} 2=\mathrm{Pa}$, vacuum is not formed and contact with the displacement plane is broken. If $0 \leq x<x$ r , then $P_{2} \leq \overline{P_{2}}$, where $\overline{P_{2}}$ is the ultimate absolute pressure, then a vacuum is formed through the operation of the turbine or turbine pump due to the action of the generated swirl flow in the capacity of the vacuum contact device, which is the capacity body of the robot. The normal contacting force of the robot housing associated with the vacuum contact device is calculated by the formula

$\mathrm{N}=(\mathrm{Pa}-\mathrm{P} 2) \cdot \mathrm{S}-\mathrm{kx}$,

where $\mathrm{S}$ is the effective area of the robot's contact with the displacement surface, $\mathrm{x}$ is the value of the variable gap between the contacting surface of the robot end face and the displacement surface, and $\mathrm{k}$ is the elastic coefficient of elastic material of the end face of the robot body.

The type of pump performance and aerodynamic drag of the contacting device, pressure versus flow rate in relative coordinates are shown in Figure. 5, a, b. Here $P_{н}, P_{у}$ and $Q_{н}, Q_{y}-$ respectively the pressure and flow rates in the cavities of the pump and vacuum contacting device; $\mathrm{P}_{\text {но }}, \mathrm{R}_{\text {уо }}$ and $\mathrm{Q}_{\text {но }}, \mathrm{Q}_{\mathrm{yо}}$ - are the average values of pressures and flows in the cavity of the pump and the vacuum contacting device.

Finding point $\mathrm{A}$ of the operating mode is shown in Figure. 6, where the dependences of pressure on flow for pump 1 and aerodynamic resistance of the contacting device 2, 3 and 4 are combined. In this case, graph 2 corresponds to the nominal operating mode, and graphs 3,4 show the deviation from the nominal mode. The inclusion of pressure feedback corresponds to the adaptive mode of operation when, as a result of controlled adaptation, schedules 3 and 4 come close to schedule 2, and their combination determines the completion of adaptation.

A similar current feedback determines the adaptation according to the force of the contact.

\section{Conclusion}

The article presents the results of studies of a vacuum device for contacting a mobile robot to a movement surface using a centrifugal pump and turbine. The vortex air motion in such vacuum contact devices and the main parameters are considered. A graphical definition of the operating modes is given, including on the basis of adaptation methods. Experimental dependences of the pressing force of the coupling device with the surface on the size of the gap between the contact surfaces in the phase of movement of the robot are obtained. The characteristics of the current strength in the excitation winding of DC motors that drive the structural elements of the vacuum generation device, depending on the size of the gap, are analyzed.

\section{Acknowledgments}

This study was supported by the Russian Foundation for Basic Research Grant 18-08-00357.

\section{References}

1. Krasnoslobodtsev V.Ya., Skvortsov V.Yu. Adaptive pneumatic vacuum grips and robot supports. St. Petersburg: Tech. University SPb., 1996, 100 p. 
2. Sensing systems and adaptive industrial robots / Ed. E.P. Popova. M .: Mechanical Engineering, 1985, $256 \mathrm{pp}$.

3. Fu G., Gonzalez R., Lee K. Robotics: World 1989.620 p.

4. Chernousko F.L., Bolotnik N.N., Gradetsky V.G., Manipulation robots. Dynamics, management, optimization. M., "Science", 1989, 363 pp.

5. Gradetsky V.G., Knyazkov M.M., Kravchuk L.N., Samokhvalov G.V., Chashchukhin V.G. Device for washing smooth surfaces. Patent No. 132327 dated 03/27/2013.

6. Darintsev O.V., Migranov A.B. Autonomous vacuum gripper microrobot. Patent RU 2266810 C1, No. 36, 2005

7. Barabanov G.P., Bogdanov S.V., Barabanov V.G. Sensitive vacuum grip. Patent RU 2283751 C1, Bull. No. 26, 2005

8. Sysoev S.N., Kuznetsov R.V., Pronin A.V., Alexandrov I.V., Pasechnik M.A. Vacuum gripping correction module. Patent RU 2431561 C2. Bull. No29, 2011

9. Longo D., Muscato G. Adhesion techniques for climbing robots: state of the art and experimental consideration // Proc. of 11th Intern. Conf. on Climbing and Walking Robots (CLAWAR-2008), 08-10 September 2008, Coimbra, Portugal.

10. Luk B., Collie A., Billinngsley J. Robug II: An intelligent wall climbing robot// Proc. IEEE Int. Conf. Robotics and Automation 1991. Vol. 3 P. 2342-2347.

11. Schmidt D., Berns K., Ohr J. Analysis of sliding suction cups for negative presure adhesion of a robot climbing on concrete walls // Adaptive Mobile Robotics. Proc. of the 15th Int. Conf. on Climbing and Walking Robots (CLAWAR-2012), 23-26 July 2012, Baltimore, USA, P. 813-820.

12. Illingworth L., Reinfeld D. Vortex attractor for planar and non-planar surfaces. U.S.A. Patent 6.619.922, Sept. 16. 2003.

13. Gradetsky V.G. Dynamic processes in miniature mobile robots with vacuum contact to moving surfaces // Bulletin of the Nizhny Novgorod University. N.I. Lobachevsky. 2011. No4. Part 2, p. 104-105.

14. Gradetsky V.G., Fomin L.F. Dynamic processes in vacuum systems of miniature mobile robots // Mechatronics, automation, control. 2013. No. 9. p. 10-14.

15. URL: www.camozzi.com

16. Tachkov A.A., Kalinichenko S.V., Malykhin A.Yu. Modeling and evaluating the effectiveness of the retention system of a small-sized autonomous robot of vertical movement with vacuum grips. // Mechatronics, automation, control. 2016. No3. p. 178-186.

17. Gradetsky V.G., Knyazkov M.M., Fomin L.F., Chashchukhin V.G. The mechanics of miniature robots. $\mathrm{M}$.: Nauka, 2010, $271 \mathrm{p}$.

18. Gradetsky V.G., Veshnikov V.B., Kalinichenko S.V., Kravchuk L.N. The controlled movement of mobile robots on randomly oriented in space surfaces. M .: Nauka, 2001, 335 p.

19. Apostolescu T.C., Udrea C., Duminica D., Iorasco C., Bogato L., Laurentiu A.C. Development of a climbing robot with vacuum attachment cups // Proceedings of International Conference MECHITECH'11, September 22-23, 2011, Bucharest, p. 258-267.

20. Watanabe M., Tsukagoshi H. Snail inspired climbing robot using fluid adhesion to travel on rough concrete walls and ceilings. // Advanced in cooperative robotics. Proceedings of CLAWAR-2016, 12-14 September 2016, London, UK, p.79-87.

21. Luk B., Collie A., Billingsley J. Robug II: An intelligent wall climbing robot // Proc. IEEE Int. Conf. Robotics and Automation. 1991. vol. 3. P. 2341-2347.

22. Semidubersky M.S. Pumps, compressors, turbines. M .: Higher school. 1966, 262 p. 
